\title{
THE FATE OF INTRA-GROUP MEDIUM
}

\author{
YUHRI ISHIMARU \\ National Astronomical Observatory, \\ 2-21-1, Mitaka, Osawa, Tokyo 181, Japan. \\ email: ishimaru@th.nao.ac.jp
}

\begin{abstract}
Combining X-ray data for clusters of galaxies, groups, and elliptical galaxies, we have obtained the evidence for that groups eject parts of the intra-group medium (IGM) via supernovae-driven 'group winds' like ellipticals. This scenario is confirmed by $1 \mathrm{D}$ hydrodynamical simulations.
\end{abstract}

\section{Elliptical-Group-Cluster sequence}

Figure 1 shows the relation between gravitational mass $M_{\text {grav }}$ and $M_{\text {gas }} / L_{B}$. While the gas mass to galaxy luminosity ratio is almost constant for rich clusters, it decreases with a reduction in gravitational mass for groups smaller than $M_{\text {grav }} \sim 510^{13} M_{\odot}$. One can clearly identify the sequence from elliptical to clusters (hereafter EGC sequence). The EGC sequence is also established in the relation between $M_{\text {grav }}$ and FeML with the same critical mass. If this correlation is due to accretions of baryons, FeMLs must take the universal value. The decreases of gas and iron suggests that smaller groups lose more intra-group medium (IGM). Especially, the EGC sequences are smoothly connected to ellipticals. Thus, this correlation can be interpreted as follows: While clusters have sufficient binding energy to keep the intra-cluster medium throughout their evolution, groups eject parts of the IGM via supernovae-driven 'group winds' like ellipticals. The efficiency of group winds should be negatively correlated with the binding energy, and therefore, the EGC sequence would emerge.

\section{Group wind scenario}

We examine that group winds can indeed reproduce the EGC sequence under the assumption of the universal initial baryon fraction, using the $1 \mathrm{D}$ 

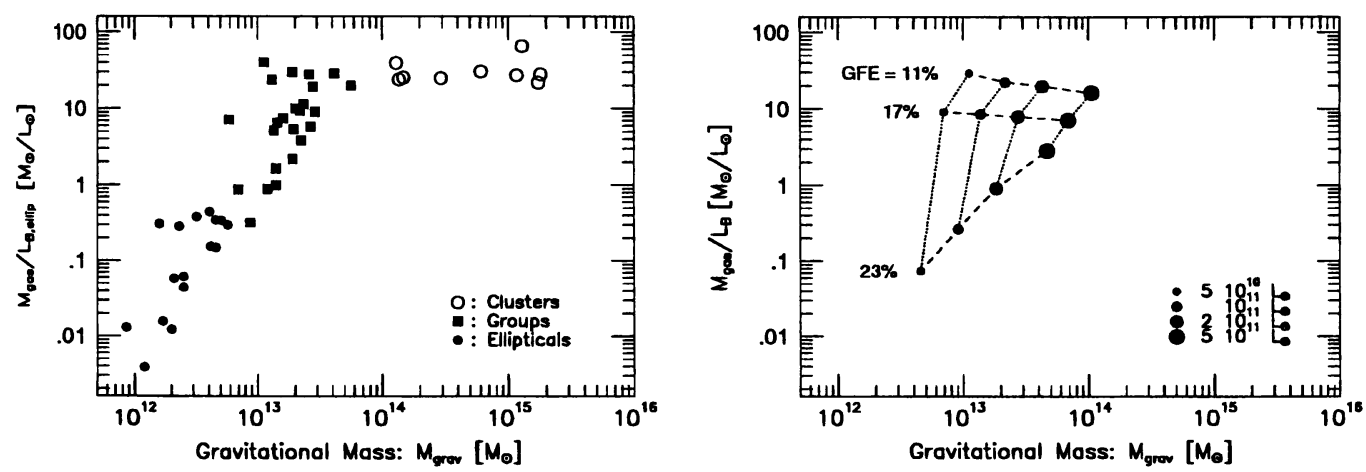

Figure 1. (left) The observed EGC sequence: The open circles, closed squares, and closed diamonds represent clusters, groups, and ellipticals, respectively.

Figure 2. (right) The calculated final gas-to-luminosity ratio as a function of gravitational mass: The total luminosities of each models are $510^{10}, 10^{11}, 210^{11}, 510^{11} L_{\odot}$, which are given by smaller to larger closed circles. The dotted and dashed lines connect models with the identical luminosities and galaxy formation efficiencies, respectively. The values of GFE are put at the side of corresponding lines.

hydrodynamical model. We apply the 1D hydrodynamical scheme for the gas flows in ellipticals (e.g., David et al. 1990; Ciotti et al. 1991) to the groups. The evolution of IGM is examined for groups with $L_{B} \sim 510^{10}$ $510^{11} L_{\odot}$. The initial galaxy fraction is determined by the galaxy formation efficiency $\mathrm{GFE}=M_{\text {galaxy }} / M_{\text {baryon }}$. The observed value of $M / L_{V} ; \sim 70-$ $150 M_{\odot} / L_{\odot}$ determines the possible range of GFE as $17 \pm 6 \%$. Figure 2 shows the relation between the final $M_{\text {gas }} / L_{B}$ ratio and total mass. As shown in this figure, a small dispersion in GFE causes a large dispersion in the efficiency of gas ejection. Because the GFE represent the energy source in a unit gas mass, groups with higher GFE eject more IGM. In addition, smaller groups is more sensitive to the GFE. Therefore, a possible dispersion in GFE easily generate the observed trend and dispersion.

If rich clusters are formed through hierarchical clusterings, the EGC sequence requires that all group wind ejecta come back successfully at merging of groups. On the other hand, if each galaxy system has evolved isolately, the EGC sequence can be understood simply by group winds. Moreover, unnatural baryon enhancement in rich clusters is not required.

\section{References}

Ciotti, L., D'Ercole, A., Pellegrini, S., \& Renzini, A. 1991, ApJ, 376, 380

David, L. P., Forman, W., \& Jones, C. 1990, ApJ, 359, 29 